\title{
Complex behavioural changes after odour exposure in Drosophila larvae
}

\author{
JULIEN COLOMB, NICOLA GRILLENZONI, REINHARD F. STOCKER \& ARIANE RAMAEKERS \\ Department of Biology, University of Fribourg
}

\begin{abstract}
A variety of odorants attract Drosophila larvae, although this behaviour can be modulated by experience. For instance, larvae pre-exposed to an attractive odorant may subsequently display less attraction towards the same compound. In previous reports, this phenomenon has been interpreted as a drop in olfactory sensitivity, caused by sensory adaptation. We tried to elucidate the basis of this behavioural modification by pre-exposing larvae to various odours. After multiple pre-exposure cycles larvae were repulsed by initially attractive odours, and pre-exposure did not change the threshold concentration driving a behavioural response. We therefore believe that sensitivity to the odorant was only slightly affected in our protocol. Our results thus do not support the previous interpretation and rather suggest that olfactory pre-exposure induces a change in the hedonic value of the odour. Although we did not succeed in elucidating the exact nature of the underlying mechanism, we can reject an association of the odour with the absence of food as an interpretation of the observed behavioural changes; this is because addition of food did not abolish the repulsion to the pre-exposed odour. In addition to ruling out previous interpretations of odour pre-exposure effects, this study stresses the complexity of Drosophila larval behaviour.
\end{abstract}

Both vertebrates and invertebrates show efficient behaviour in response to biologically relevant olfactory signals. They are able to extract odours related to food, dangerous conditions or mates from a highly complex chemical environment. Accordingly, their sensitivity to background odours is subject to modification, acting mainly through the mechanism of olfactory adaptation. In addition, the actual meaning of an odour is not rigidly programmed, but depends on its context and may change over time. Therefore, the olfactorily driven behaviours of animals tend to adapt to the local environment, notably by olfactory learning. These behavioural modifications, albeit well defined in human psychophysiological assays, are difficult to identify in experiments involving animal models.

Olfactory adaptation is defined by psychophysiologists as a reduction in sensitivity to an odour after repeated or

Correspondence and present address: J. Colomb, Gènes at Dynamique des Systèmes de Mémoire, CNRS UMR 7637, ESPCI, 10 rue Vauquelin, 75005 Paris, France (email: julien.colomb@espci.fr). N. Grillenzoni, R. F. Stocker and A. Ramaekers are at the Department of Biology, University of Fribourg, CH-1700 Fribourg, Switzerland. prolonged exposure to that same odour (Dalton 2000). This definition comprises both olfactory adaptation and habituation as defined by Bernhard \& van der Kooy (2000), and gives no indication about its cellular basis, that is, sensory adaptation (Zufall \& Leinders-Zufall 2000) or central habituation (Wilson 2000). Different properties of olfactory adaptation behaviour have been highlighted. For instance, the degree of adaptation depends on the intensity of the odorant during pre-exposure, and is odorant specific. Indeed, odorant specificity has been used to test discriminative ability in Drosophila: a decrease in the response to an odour B after pre-exposure to an odour A has been interpreted as an incomplete discrimination of the two odours (Cobb \& Domain 2000; Boyle \& Cobb 2005).

Olfactory learning has been studied intensively, in particular in the context of classical conditioning in both vertebrates and invertebrates, using many different approaches (reviewed in Milner et al. 1998; Davis 2005). For instance, in Drosophila larvae, olfactory or visual cues (CS; conditioned stimulus) become more attractive after association with a pleasant gustatory stimulus (US; unconditioned stimulus; Scherer et al. 2003; Gerber et al. 2004; 
Honjo \& Furukubo-Tokunaga 2005). In some cases, the new behaviour resulting from the association between a CS and a US can be elicited by another stimulus, $\mathrm{CS}^{\prime}$, similar to the CS. This phenomenon, called generalization, has been used to measure similarity between different and often discriminated stimuli (Ghirlanda \& Enquist 2003; Wright \& Smith 2004; Guerrieri et al. 2005).

The processes of adaptation and learning (i.e. the loss of sensitivity and a change in hedonic value, respectively) are theoretically clearly distinct, but are empirically difficult to separate. For instance, olfactory adaptation is commonly tested by comparing the olfactory responses of animals pre-exposed to the odorant with the responses of control animals. A lower response is interpreted as a reduced sensitivity to the odour, reflecting olfactory adaptation. However, this lower response could also indicate that the animal values the odour as less positive. Such an effect was demonstrated in a study on Caenorhabditis elegans where pre-exposure to an odorant in the absence of food, a protocol previously thought to lead to olfactory adaptation (Colbert \& Bargmann 1995), was shown to lead to olfactory associative learning (Nuttley et al. 2002). In this situation, the absence of food acts as a negative US associated with the odorant, leading to a decrease in the chemotactic response towards that odorant.

Drosophila has been used for decades to decode the neural and genetic basis of behaviour. Since the olfactory system of larvae is organized similarly to the adult one despite its limited number of odorant receptor neurons (Kreher et al. 2005; Ramaekers et al. 2005), the fruit fly larva appears to be a promising model system to study olfactory processing. There is evidence for olfactory associative learning in Drosophila larvae (Scherer et al. 2003; Hendel et al. 2005; Honjo \& Furukubo-Tokunaga 2005). On the other hand, Cobb \& Domain (2000) and Boyle \& Cobb (2005) used olfactory adaptation of larvae to test olfactory discrimination and, accordingly, proposed models of peripheral olfactory coding. Wuttke \& Tompkins (2000) tested larvae mutant for trp, a gene encoding a calcium channel whose expression is required during development for olfactory adaptation in the adult (Störtkuhl et al. 1999). They observed no effect of trp loss of function in their experimental set-up. However, Wuttke \& Tompkins (2000) assumed that only olfactory adaptation was modifying larval behaviour, and did not test for the presence of different forms of learning.

We investigated the mechanisms underlying behavioural changes in Drosophila larvae after pre-exposure to odorants, using a modified protocol from Cobb \& Domain (2000). We analysed our data in the context of sensory adaptation, increase in sensitivity and associative learning.

\section{GENERAL METHODS}

\section{Stocks}

Flies from a Canton-S strain (provided by T. Préat, ESPCI, Paris, France) were reared on standard corn food medium at $25^{\circ} \mathrm{C}$ on a $12: 12 \mathrm{~h}$ light:dark cycle.

\section{Odorants}

Butanol (Fluka cat. 19420, Buchs, Switzerland), hexanol (Fluka cat. 52828), nonanol (Fluka cat. 74278), ethyl caproate (Aldrich/Sigma cat. 14.896-2, Buchs, Switzerland) and ethyl acetate (Merck Schweiz cat. 109623.1000, Dietikon, Switzerland) were used, all highest purity grade. Odorants were displayed on filter paper discs $10 \mathrm{~mm}$ in diameter (Schleicher and Schuell cat. 589/2, Bottmingen, Switzerland).

\section{Behavioural Tests}

We carried out experiments on agar plates consisting of petri dishes $85 \mathrm{~mm}$ in diameter without ergot (Greiner/ Huber cat. 632180, Reinach, Switzerland) covered with 2.5\% Select Agar (Invitrogen/Lubio Science cat. 30391023, Lucerne, Switzerland). Sugar and dry yeast plates were covered with 1\% Select Agar containing 0.5\% autolysed yeast (DIFCO/VWR International cat. 0229-17-6, Dietikon, Switzerland) and 7.5\% sugar (from a local grocery store). Yeast plates were produced by covering the surface of the standard agar plates with fresh baking yeast (from a local grocery store) soaked with distilled water. We used young third-instar larvae (75 $\pm 3 \mathrm{~h}$ after egg laying). As no difference was seen between tests done in the morning or afternoon, we pooled all data. Control and experimental groups were always tested in parallel, using larvae from the same culture bottle.

Larvae were washed from the food with $17 \%$ sucrose solution. After three rinses in tap water, about 50 larvae were put in a petri dish for 5-15 min. They were then transferred to a pre-exposure plate that contained either an odorant (pre-exposed group) or water (control group) spread on four $10-\mathrm{mm}$ filter paper disks. Filter papers were evenly spaced along the edge of the plate, placed on the agar surface. The amount of odorant indicated below, for each experiment, for the pre-exposure plates relates to the total, i.e. $10 \mu \mathrm{l}$ corresponds to $4 \times 2.5 \mu \mathrm{l}$. Larvae were pre-exposed in the dark for $10 \mathrm{~min}$ in a switchedoff incubator at room temperature under a fume hood. Then, they were transferred into a clean agar plate for a rest period of $10 \mathrm{~min}$ under the fume hood in the presence of light. We carried out this procedure, $10 \mathrm{~min}$ of preexposure and $10 \mathrm{~min}$ of rest, either once or three times.

We carried out the tests as described previously (Heimbeck et al. 1999). Briefly, we placed larvae in the middle of an agar plate containing a pair of filter paper disks on opposite sides, soaked, respectively, with odorant and water. The odorant was put randomly on the left or the right side of the plate. The test plates were then placed under a cardboard cache, in a fume hood. After $5 \mathrm{~min}$, we took a picture of each test plate and counted the larvae. A response index (RI) was calculated: RI $=\left(N_{\mathrm{s}}-N_{\mathrm{c}}\right) /\left(N_{\mathrm{s}}+N_{\mathrm{c}}\right)$. $N_{\mathrm{s}}$ represents the number of larvae at a distance $d \leq 30 \mathrm{~mm}$ from the odour source. $N_{\mathrm{c}}$ is the number of larvae found inside an identical surface on the opposite side. Positive and negative RIs reflect attraction and avoidance, respectively, and $\mathrm{RI}=0$ indicates indifferent behaviour (tested by measuring attraction towards water). Data presented in the same graph were always from experiments 
done in parallel. The proportion of larvae found in the neutral area between disks was constant between groups; we discarded the data if this proportion was higher than $40 \%$ (limit chosen arbitrarily before doing the experiments).

Odorants were diluted in water. Since the chemicals were soaked on wet filter paper, even pure odorants were actually diluted in water; we can thus assume that vapour concentration is directly proportional to the corresponding volume of pure soaked odorant (Cometto-Muniz et al. 2003).

\section{Statistics}

For group comparison, we used the arcsine transformation of the proportion of larvae moving to the odorant $[A=A$ sine(square $\operatorname{root}(R)) \quad$ where $\quad R=N_{\mathrm{s}} /\left(N_{\mathrm{s}}+N_{\mathrm{c}}\right)=$ $(\mathrm{RI}+1) / 2)]$. The value $(A)$ was the dependent variable of a univariate test (two tailed), weighted by the total number of choosers $\left(T=N_{\mathrm{s}}+N_{\mathrm{c}}\right)$; in cases where the side containing the odorant (left or right side of the plate) had an effect, this information was added as a fixed factor. For comparison between more than two groups, we used a Tukey post hoc test. A difference between two groups was always confirmed by a two-tailed Mann-Whitney $U$ test with $P<0.01$.

A significant difference from $\mathrm{RI}=0$ was assessed with a one-sample $t$ test. The statistical tests were carried out and plots were generated with SPSS for Macintosh version 11 (SPSS Suisse SA, 8044 Zürich, Switzerland).

\section{NO OLFACTORY ADAPTATION?}

\section{Experiment 1}

To measure olfactory adaptation, we used a protocol modified from Cobb \& Domain (2000). Briefly, we tested the behavioural effect of odour pre-exposure by comparing the olfactory response of larvae pre-exposed to an odorant with the response of larvae pre-exposed to water. We measured this response by calculating the proportion of larvae moving towards or away from an odorant (chemotaxis); the results are depicted as a response index (RI) ranging from -1 (total repulsion) to +1 (total attraction). Whereas Cobb \& Domain pre-exposed the larvae for $1 \mathrm{~h}$, we used a 10-min pre-exposure period and we allowed the larvae to rest for another 10 min before testing them (for details, see General Methods).

As pretests, we compared the response to butanol of three groups of larvae: larvae kept before the tests for $10 \mathrm{~min}$ in a normal agar plate, larvae kept for $10 \mathrm{~min}$ in an empty plate and larvae that were not handled before the tests (naïve larvae). The first group had a higher RI than the other two groups (normal agar plate: $\bar{X} \pm \mathrm{SEM}=0.85 \pm 0.2$; empty plate: $0.55 \pm 0.6$; naïve: $0.59 \pm 0.4$ ). Handling of larvae therefore did not appear to have any effect per se, but a contextual effect is possible.

\section{Results}

Figure 1a shows that control larvae were attracted by ethyl acetate $(1 \mu \mathrm{l})$, whereas larvae pre-exposed for $10 \mathrm{~min}$ to $4 \mu \mathrm{l}$ of this compound had RIs that were reduced by $85 \%$, to a level not significantly different from $0(P>0.2)$.

\section{Experiment 2}

In previous studies, results comparable to those of experiment 1 were interpreted as olfactory adaptation (Störtkuhl et al. 1999; Cobb \& Domain 2000; Wuttke \& Tompkins 2000). However, an alternative hypothesis was not addressed in these studies, i.e. that the larvae may have associated the odorant with an unidentified negative US. To discriminate between these two interpretations, we carried out two more experiments (experiments 2 and 3).

In experiment 2 , the larvae were exposed three times to ethyl acetate or butanol. If associative learning indeed occurred, repeating the pre-exposure to the odorant should increase the strength of the association between the odorant (which corresponds to the CS) and the unidentified negative US (Rescorla \& Wagner 1972). Hence, negative RIs are predicted. In contrast, if the changes were due to olfactory adaptation only, further pre-exposure cycles would lead to an RI closer to 0.

\section{Results}

After three cycles of pre-exposure to ethyl acetate, larvae showed a lower score than those exposed only once and indeed avoided the odour $(P<0.001)$, whereas control larvae were still normally attracted (Fig. 1a). Larvae preexposed one or three times to $10 \mu \mathrm{l}$ of butanol were repelled by butanol $(2.5 \mu \mathrm{l})$, whereas control larvae were strongly attracted (Fig. 1b). Hence the results were not specific to ethyl acetate.

\section{Experiment 3}

In experiment 3, we tested the effect of pre-exposure to two repelling odorants: nonanol (Cobb \& Domain 2000) at a concentration of $2.5 \mu \mathrm{l}$ and ethyl caproate at $0.5 \mu \mathrm{l}$ which we found to be strongly repulsive for larvae (A. Ramaekers, unpublished data). If pre-exposure was associated with olfactory adaptation, RIs would be expected to be closer to 0 than those of the controls. On the other hand, if pre-exposure leads to an association between the repelling odorant (CS) and a negative US, one should observe an increase in avoidance, i.e. more negative RIs than the controls.

\section{Results}

With both odorants, the RIs of control and pre-exposed larvae were indistinguishable even with a very high sample size (nonanol). This indicates that pre-exposure had no effect on larval olfactory responses to these odorants (Fig. 1c, d).

\section{STIMULUS STRENGTH AND SPECIFICITY}

The reduction in olfactory attraction from adaptation depends on both stimulus strength and stimulus specificity (Dalton 2000). Nevertheless, such dependence is also 


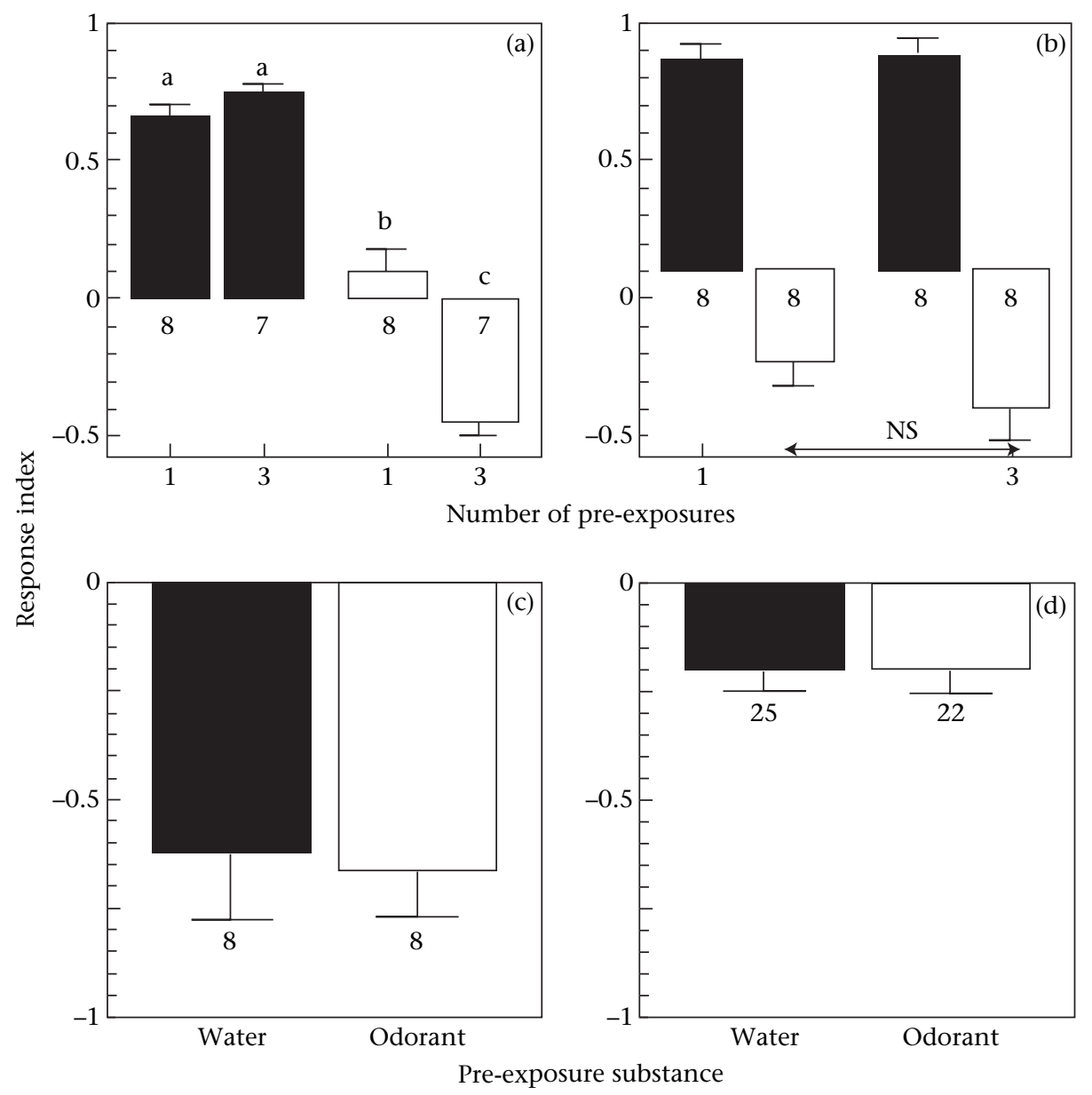

Figure 1. Olfactory responses of Drosophila larvae pre-exposed to water ( $\square$ ) or to an odorant $(\square)$ for 10 min or $3 \times 10$ min, as indicated. For details of the response index, see text; a positive index indicates attraction, a negative one repulsion. The concentrations used in pre-exposure were four times higher than those used for the tests. (a) $1 \mu$ l of ethyl acetate. Letters represent different groups of data significantly different from each other at $P<0.01$. (b) $2.5 \mu \mathrm{l}$ of butanol; positive and negative response indices are significantly different. (c) $0.5 \mu \mathrm{l}$ of ethyl caproate or (d) $2.5 \mu \mathrm{l}$ of nonanol after $10 \mathrm{~min}$ pre-exposure. Means \pm 1 SEM are shown. Numbers of independent tests involving about 50 larvae each are also shown.

a characteristic of other learning mechanisms, such as classical conditioning (Ghirlanda \& Enquist 2003; Wright \& Smith 2004). In previous studies (Störtkuhl et al. 1999; Cobb \& Domain 2000; Wuttke \& Tompkins 2000; Fletcher $\&$ Wilson 2002), researchers interpreted such observations as evidence for the presence of adaptation in their experimental set-up. To examine this interpretation, we tested whether those characteristics also applied to our protocol, although our protocol does not solely involve adaptation. We tested the response of larvae towards $2.5 \mu$ of butanol after pre-exposure to increasing concentrations of this odorant. To investigate the role of stimulus specificity on the behavioural change, we tested the response towards butanol $(2.5 \mu \mathrm{l})$ after pre-exposure to different odorants.

\section{Results}

We found a negative correlation between the proportion of larvae approaching the odorant and the concentration of odorant during the pre-exposure (covariate analysis: $F_{1,29}=63.7, P<0.001$; Fig. $2 \mathrm{a}$ ). When preexposed to $10 \mu \mathrm{l}$ of butanol, larvae subsequently avoided the odorant, whereas they showed a null RI when preexposed to smaller concentrations of this compound.

Pre-exposure to ethyl acetate had no or little effect on the attraction towards butanol $(P=0.059$; Fig. $2 b)$, suggesting that the drop in RI following pre-exposure was indeed stimulus specific. In contrast, the response towards butanol of larvae pre-exposed to hexanol (another aliphatic alcohol) was lower than the RI of control larvae pre-exposed to water $(P<0.001)$, and higher than the RI of larvae pre-exposed to butanol ( $P=0.026$; Fig. $2 b)$.

\section{INCREASE IN OLFACTORY SENSITIVITY?}

Studies on human olfaction have shown that exposure to an odorant can sometimes increase olfactory sensitivity (Dalton 1996) and that this effect may be the result of peripheral mechanisms (Yee \& Wysocki 2001). If higher concentrations of odorant become aversive, such an effect could explain our results, as proposed by Boyle \& Cobb (2005). We therefore investigated this hypothesis, by testing the responses of larvae towards different concentrations of ethyl acetate, after pre-exposure to $4 \mu \mathrm{l}$ of either 

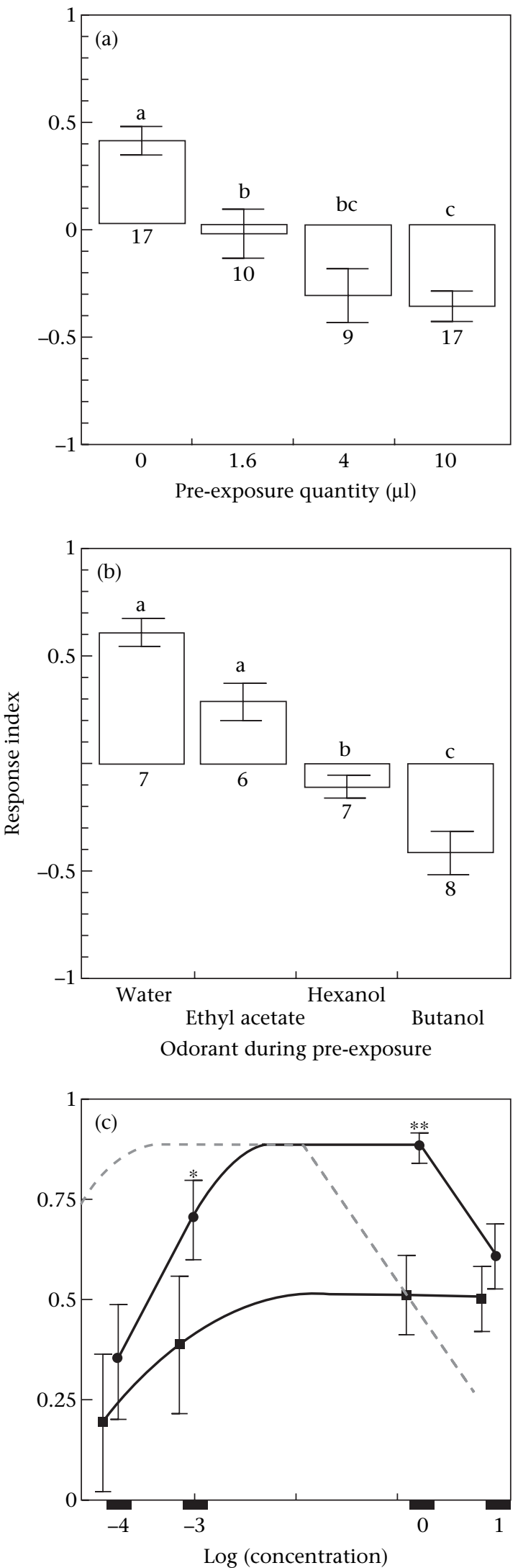

Figure 2. Olfactory responses towards $(\mathrm{a}, \mathrm{b})$ butanol $(2.5 \mu \mathrm{l})$ and $(\mathrm{c})$ ethyl acetate (different concentrations) after 10-min pre-exposure to $(a, c)$ different quantities of the same odorant or (b) different odours this compound or water. We chose to test responses towards ethyl acetate because this odorant appears to be repulsive at high concentration. If an effect of exposure on sensitivity shifts the dose-response curve to the left (according to the dashed line in Fig. 2c) as proposed by Boyle \& Cobb (2005), the detection threshold should move towards a lower concentration.

\section{Results}

The results did not fit with this prediction. We rather observed that the response of pre-exposed larvae followed the curve of the control larvae, albeit with a lower amplitude (Fig. 2c). In particular, it appeared that the detection threshold remained stable. Thus, our results argue against the presence of an effect of pre-exposure on sensitivity.

\section{EFFECTS OF ADDING FOOD AND WATER}

The observed odour avoidance behaviour of pre-exposed larvae suggests that associative learning might be involved in our experimental set-up. In an attempt to test this hypothesis, we tried to identify the negative US that might be causing the drop in olfactory responses after pre-exposure. Indeed, in a similar paradigm, Nuttley et al. (2002) showed that $C$. elegans associates odorants with the absence of food which acts as a negative US. To determine whether Drosophila larvae form similar associations, we pre-exposed larvae in agar plates containing food, by adding sucrose and dry yeast to the agar or by spreading fresh baking yeast on the agar. Such 'fresh yeast plates' are known to drive larval foraging behaviour (Pereira et al. 1995). Because learning performance can drop with a change in context between learning and test phases (Haney \& Lukowiak 2001; Law et al. 2004), pre-exposure and tests were made in the same type of plates.

\section{Results}

The addition of sucrose and dry yeast to the agar plates did not change the behaviour of control or of pre-exposed larvae (Fig. 3a). When we used fresh yeast, the larvae tended to cluster on the yeast (data not shown), indicating that they recognized it as a food source. Figure $3 b$ shows that the responses of all groups to butanol were closer to 0 in yeast plates. A straightforward explanation could be that odour or taste of the yeast distracted larvae from responding to butanol during the test but had no effect during pre-exposure. The fact that the differences in the absolute RI values between normal and yeast plates were similar

or water. For details of the response index, see text; a positive index indicates attraction, a negative one repulsion. In (c), 0 on the abscissa represents $1 \mu \mathrm{l}$ of pure odorant (the same condition as in Fig. 1a), $N \geq 8$. : Control; $\mathbf{\square}$ : pre-exposed to $4 \mu \mathrm{l}$ of pure ethyl acetate; - - -: theoretical sensitization curve. Letters represent data significantly different at $P<0.05$; ' $b c^{\prime}$ means that the difference between this group is not significantly different from either $b$ or $c$, but is different from $a$. Means \pm 1 SEM are shown. Numbers of independent tests involving about 50 larvae each are also shown. ${ }^{*} P<0.05 ;{ }^{* *} P<0.01$. 


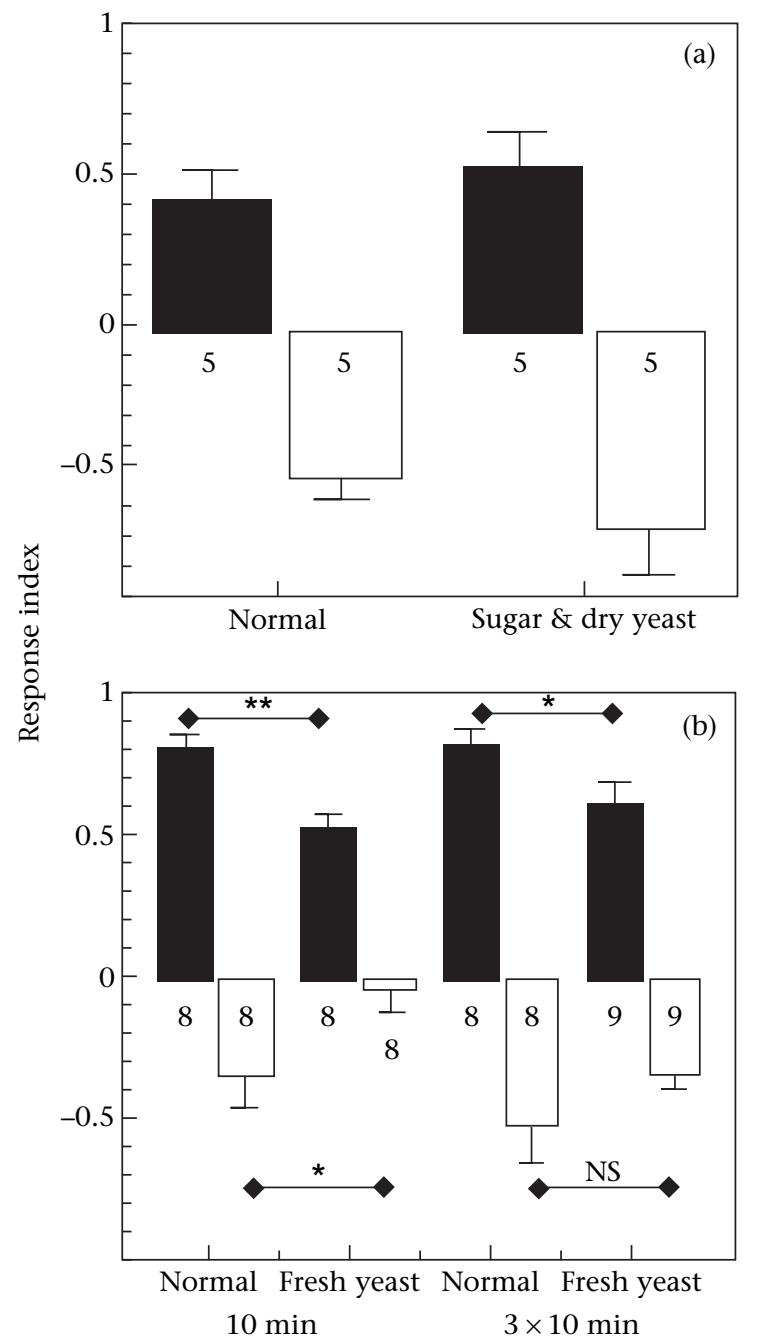

Figure 3. Olfactory responses to butanol after pre-exposure to water ( $\boldsymbol{\square}$ ) or butanol $(\square)$, in different types of plates (pre-exposure and tests were carried out in the same type of plates; see General Methods). For details of the response index, see text; a positive index indicates attraction, a negative one repulsion. (a) Sugar and dry yeast in the agar; 10-min pre-exposure. (b) Comparison of experiments on normal plates or in the presence of fresh yeast, with pre-exposure for $10 \mathrm{~min}$ or $3 \times 10 \mathrm{~min}$, as indicated. Means \pm 1 SEM are shown. Numbers of independent tests involving about 50 larvae each are also shown. ${ }^{\star} P<0.05,{ }^{* *} P<0.01$. See text for further details.

for control and pre-exposed larvae (Fig. 3b, and data not shown) argues in favour of this hypothesis. Furthermore, after three cycles of exposure to butanol in yeast plates, larvae were actively repelled by butanol $(P<0.01)$.

Additional experiments in which the amount of water was increased in the plates did not affect behaviour (data not shown), suggesting that dryness does not represent a negative US either.

\section{DISCUSSION}

\section{Intact Odour Sensitivity}

Similar to previous studies (Cobb \& Domain 2000; Wuttke \& Tompkins 2000; Boyle \& Cobb 2005; Michels et al. 2005), we observed that larvae modify their response to an odorant after pre-exposure to the same compound. We also confirmed that this modification is stimulus specific and dependent upon the stimulus strength during pre-exposure. In particular, the positive (attractive) response to ethyl acetate dropped almost to 0 after one pre-exposure cycle. Olfactory adaptation was previously considered as the psychological basis of such observation. In this study, we questioned this interpretation. In particular, by increasing the number of pre-exposure cycles from one to three, we found that the larval response to ethyl acetate was converted from attraction to avoidance. In addition, in the case of butanol, one pre-exposure cycle was sufficient to induce this inversion of behaviour. This phenomenon had already been measured with octanol (Cobb \& Domain 2000), and was further investigated recently using 1-h pre-exposure (Boyle \& Cobb 2005). The two protocols appear to produce qualitatively similar data which might thus be the result of the same mechanisms. Our results suggest that the difference between the responses to ethyl acetate measured after one and three pre-exposure cycles in our study was quantitative rather than qualitative. The RI close to 0 measured after one pre-exposure cycle to ethyl acetate could correspond to a partial inversion of the olfactory behaviour rather than a decrease in sensitivity, as was proposed by Cobb \& Domain (2000), Wuttke \& Tompkins (2000) and Boyle $\&$ Cobb (2005).

However, our observations could still be explained on the basis of adaptation. As proposed by Cobb \& Domain (2000), an odorant would elicit responses in both 'attraction' and 'repulsion' receptor neurons (mediating attractive or repulsive behaviour, respectively). The latter would adapt more slowly, being the only cells that remain firing after pre-exposure. Consequently, a partial loss of sensitivity would lead to repulsion. This model predicts that, with further exposure, the response becomes extinct. In contrast to this prediction, we observed that additional periods of pre-exposure did not reduce the avoidance but, on the contrary, tended to lead to more negative RIs. Therefore, we propose that the change from attraction to repulsion after several cycles of pre-exposure might be the result of mechanisms different from adaptation.

Previous researchers also interpreted their findings in terms of olfactory adaptation because the behavioural modifications depended on stimulus intensity and stimulus specificity, which are typical properties of olfactory adaptation (Cobb \& Domain 2000; Dalton 2000; Wuttke $\&$ Tompkins 2000). However, we question this assumption, since both properties also apply to our protocol, which we believe to be largely independent of adaptation mechanisms. Hence, other learning processes are characterized by a dependence on both stimulus concentration and stimulus specificity. This is, for instance, the case for classical conditioning (Wright \& Smith 2004).

\section{Hypotheses to be Ruled Out}

At first, we considered whether nonassociative mechanisms such as sensitization could be involved in the 
observed behavioural modifications. If sensitization was induced by pre-exposure to the odorant, one should observe a subsequent increment in response compared to the control response, without an effect on sensitivity. This hypothesis is obviously not compatible with our results since pre-exposure led to a decrease in attraction or even a shift towards avoidance, of the olfactory responses.

An alternative hypothesis presented by Boyle \& Cobb (2005) predicts that pre-exposure would increase the sensitivity to the odorant. In this case, a concentration $c$ of the odorant would be perceived as if a concentration $d>c$ had been presented (Dalton 1996), and the animals would behave accordingly: the dose-response curve would be shifted towards lower concentrations (see dashed line in Fig. 2c). Since, as for adults, attractive odorants might become repulsive to larvae at high concentrations (Fig. 2c; see also Boyle \& Cobb 2005), one could predict that, after pre-exposure, a given concentration eliciting an attractive response in the control conditions would become repulsive. However, our results did not confirm the shift to lower concentrations of the doseresponse curve after pre-exposure predicted by this model. Furthermore, Boyle \& Cobb (2005) strikingly observed no increase in the attractiveness of any odour after prestimulation with the same or a different odorant; their results therefore do not fit with this hypothesis either.

Finally, we tested the hypothesis of a classical conditioning. If larvae indeed associated odorants with the absence of food, as do nematodes (Nuttley et al. 2002), addition of yeast to the pre-exposure plates should have reduced or abolished the behavioural modification (Annau \& Kamin 1961). In contrast, our experiments suggest that they still learn in the presence of food (Fig. 3). Thus, we conclude that the absence of food cannot be a major US, since its suppression does not eliminate the behavioural modification.

Dryness could be ruled out as a US as well, since the addition of water in the pre-exposure agar plate had no effect on the behavioural modifications. According to another report (Dukas 1999), the manipulation of larvae per se may play the role of a negative US. However, in our protocol, larvae were also manipulated before having contact with the odorant. Consequently, the odour could not be a good predictor of this putative US and should not become associated with it (Rescorla 1968).

Bright light is an aversive stimulus for early third-instar larvae (Gerber et al. 2004) and so might act as a US. However, since we kept the larvae in the dark during pre-exposure and in the light during the rest period, a reinforcing effect of light would act against the observed behaviour.

Boyle \& Cobb (2005) obtained similar results to ours, although in their set-up larvae were prevented from having direct (gustatory) contact with the odorant; this contact thus appears not to be required for the behavioural change. However, a deleterious effect of high odorant concentrations could still act as the US. Assuming that the noxious effect depends on odorant concentration, smaller doses of odorant should be correlated with a weaker US. Hence, this hypothesis predicts that pre-exposure to decreasing concentrations of odorant should result in smaller behavioural changes. This is indeed what we observed in the case of butanol (Fig. 2a).

\section{Fixed Hedonic Value for Repellent Odorants?}

The fact that the response to nonanol and ethyl caproate did not change after pre-exposure is striking. While this result is difficult to interpret, it indicates that, in the case of these repulsive odorants, there is no evidence for the presence of olfactory adaptation or olfactory learning. We propose that these odorants may already possess an innate and fixed hedonic value that could not be modified by our set-up. When Honjo \& Furukubo-Tokunaga (2005) tried to associate an odour (CS) with sucrose (US), they found that this association was possible only when using certain odorants and not others. Their results show that larvae can react differently to olfactory associative learning, depending on the odorant chosen as the CS. Our observations provide another example of this striking but poorly documented effect of odorant identity on olfactory learning (see also Keene et al. 2004).

\section{Concluding Remarks}

Although the interpretation of our results remains largely open, we have shown that olfactory adaptation is not sufficient to explain the behavioural modifications provoked by pre-exposure to an odorant. Our results also do not fit with an increase in odour sensitivity as proposed by Boyle \& Cobb (2005). Our simplest working model involves an associative learning process. However, we were not able to confirm this hypothesis, because we did not succeed in identifying a negative US. Alternatively, different mechanisms could act in parallel, or other nonassociative learning mechanisms are conceivable (e.g. disinterest of larvae for a previously experienced odorant).

Drosophila larvae are often considered to be continuous feeders with a limited behavioural repertoire. In particular, their olfactory system, characterized by no more than 21 receptor neurons, is thought to be rudimentary. In contrast, this study and others (Scherer et al. 2003; Gerber et al. 2004; Honjo \& Furukubo-Tokunaga 2005) stress the complexity and plasticity of larval olfactorily driven behaviour which could mirror the importance of odour perception for larval survival. Together with evidence demonstrating adult-like connectivity in the larval olfactory system (Ramaekers et al. 2005), they suggest that olfactory cues may be much more crucial for larval survival than previously assumed.

\section{Acknowledgments}

We thank Dr T. Préat for sharing his CS flies, Dr F. Mery, Fribourg, for help with statistical analysis and Dr B. Gerber, Würzburg, and the anonymous referees for comments on the manuscript. This work was supported by the Swiss National Funds (grants no. 31-63447.00, 3100A0105517 to R.F.S. and 3234-069273.02 to A.R.). 


\section{References}

Annau, Z. \& Kamin, L. J. 1961. The conditioned emotional response as a function of intensity of the US. Journal of Comparative and Physiological Psychology, 54, 428-432.

Bernhard, N. \& van der Kooy, D. 2000. A behavioral and genetic dissection of two forms of olfactory plasticity in Caenorhabditis elegans: adaptation and habituation. Learning and Memory, 7, 199-212.

Boyle, J. \& Cobb, M. 2005. Olfactory coding in Drosophila larvae investigated by cross-adaptation. Journal of Experimental Biology, 208, 3483-3491.

Cobb, M. \& Domain, I. 2000. Olfactory coding in a simple system: adaptation in Drosophila larvae. Proceedings of the Royal Society of London, Series B, 267, 2119-2125.

Colbert, H. A. \& Bargmann, C. I. 1995. Odorant-specific adaptation pathways generate olfactory plasticity in C. elegans. Neuron, 14, 803-812.

Cometto-Muniz, J. E., Cain, W. S. \& Abraham, M. H. 2003. Quantification of chemical vapors in chemosensory research. Chemical Senses, 28, 467-477.

Dalton, P. 1996. Odor perception and beliefs about risk. Chemical Senses, 21, 447-458.

Dalton, P. 2000. Psychophysical and behavioral characteristics of olfactory adaptation. Chemical Senses, 25, 487-492.

Davis, R. L. 2005. Olfactory memory formation in Drosophila: from molecular to systems neuroscience. Annual Review of Neuroscience, 28, 275-302.

Dukas, R. 1999. Ecological relevance of associative learning in fruit fly larvae. Behavioral Ecology and Sociobiology, 19, 195-200.

Fletcher, M. L. \& Wilson, D. A. 2002. Experience modifies olfactory acuity: acetylcholine-dependent learning decreases behavioral generalization between similar odorants. Journal of Neuroscience, 22, RC201.

Gerber, B., Scherer, S., Neuser, K., Michels, B., Hendel, T., Stocker, R. F. \& Heisenberg, M. 2004. Visual learning in individually assayed Drosophila larvae. Journal of Experimental Biology, 207, 179-188.

Ghirlanda, S. \& Enquist, M. 2003. A century of generalization. Animal Behaviour, 66, 15-36.

Guerrieri, F., Schubert, M., Sandoz, J. C. \& Giurfa, M. 2005. Perceptual and neural olfactory similarity in honeybees. Public Library of Science, Biology, 3, e60.

Haney, J. \& Lukowiak, K. 2001. Context learning and the effect of context on memory retrieval in Lymnaea. Learning and Memory, 8, 35-43.

Heimbeck, G., Bugnon, V., Gendre, N., Häberlin, C. \& Stocker, R. F. 1999. Smell and taste perception in Drosophila melanogaster larva: toxin expression studies in chemosensory neurons. Journal of Neuroscience, 19, 6599-6609.

Hendel, T., Michels, B., Neuser, K., Schipanski, A., Kaun, K., Sokolowski, M. B., Marohn, F., Michel, R., Heisenberg, M. \& Gerber, B. 2005. The carrot, not the stick: appetitive rather than aversive gustatory stimuli support associative olfactory learning in individually assayed Drosophila larvae. Journal of Comparative Physiology, A, 191, 265-279.

Honjo, K. \& Furukubo-Tokunaga, K. 2005. Induction of cAMP response element-binding protein-dependent medium-term memory by appetitive gustatory reinforcement in Drosophila larvae. Journal of Neuroscience, 25, 7905-7913.

Keene, A. C., Stratmann, M., Keller, A., Perrat, P. N., Vosshall, L. B. \& Waddell, S. 2004. Diverse odor-conditioned memories require uniquely timed dorsal paired medial neuron output. Neuron, 44, 521-533.

Kreher, S. A., Kwon, J. Y. \& Carlson, J. R. 2005. The molecular basis of odor coding in the Drosophila larva. Neuron, 46, 445-456.

Law, E., Nuttley, W. M. \& van der Kooy, D. 2004. Contextual taste cues modulate olfactory learning in $C$. elegans by an occasionsetting mechanism. Current Biology, 14, 1303-1308.

Michels, B., Diegelmann, S., Tanimoto, H., Schwenkert, I., Buchner, E. \& Gerber, B. 2005. A role for Synapsin in associative learning: the Drosophila larva as a study case. Learning and Memory, 12, 224-231.

Milner, B., Squire, L. R. \& Kandel, E. R. 1998. Cognitive neuroscience and the study of memory. Neuron, 20, 445-468.

Nuttley, W. M., Atkinson-Leadbeater, K. P. \& van der Kooy, D. 2002. Serotonin mediates food-odor associative learning in the nematode Caenorhabditis elegans. Proceedings of the National Academy of Sciences, U.S.A., 99, 12449-12454.

Pereira, H. S., MacDonald, D. E., Hilliker, A. J. \& Sokolowski, M. B. 1995. Chaser (Csr), a new gene affecting larval foraging behavior in Drosophila melanogaster. Genetics, 141, 263-270.

Ramaekers, A., Magnenat, E., Marin, E. C., Gendre, N., Jefferis, G. S., Luo, L. \& Stocker, R. F. 2005. Glomerular maps without cellular redundancy at successive levels of the Drosophila larval olfactory circuit. Current Biology, 15, 982-992.

Rescorla, R. A. 1968. Probability of shock in the presence and absence of CS in fear conditioning. Journal of Comparative and Physiological Psychology, 66, 1-5.

Rescorla, R. A. \& Wagner, A. R. 1972. A theory of pavlovian conditioning: variations in the effectiveness of reinforcement and non reinforcement. In: Classical Conditioning. II: Current Research and Theory (Ed. by A. H. Black \& W. T. Prokasy), pp. 64-69. New York: Appleton-Century-Crofts.

Scherer, S., Stocker, R. F. \& Gerber, B. 2003. Olfactory learning in individually assayed Drosophila larvae. Learning and Memory, 10, 217-225.

Störtkuhl, K. F., Hovemann, B. T. \& Carlson, J. R. 1999. Olfactory adaptation depends on the Trp Ca2+ channel in Drosophila. Journal of Neuroscience, 19, 4839-4846.

Wilson, D. A. 2000. Comparison of odor receptive field plasticity in the rat olfactory bulb and anterior piriform cortex. Journal of Neurophysiology, 84, 3036-3042.

Wright, G. A. \& Smith, B. H. 2004. Different thresholds for detection and discrimination of odors in the honey bee (Apis mellifera). Chemical Senses, 29, 127-135.

Wuttke, M. S. \& Tompkins, L. 2000. Olfactory adaptation in Drosophila larvae. Journal of Neurogenetics, 14, 43-62.

Yee, K. K. \& Wysocki, C. J. 2001. Odorant exposure increases olfactory sensitivity: olfactory epithelium is implicated. Physiology and Behavior, 72, 705-711.

Zufall, F. \& Leinders-Zufall, T. 2000. The cellular and molecular basis of odor adaptation. Chemical Senses, 25, 473-481. 